\title{
ILAC released document on policy for uncertainty in calibration
}

Recently the International Laboratory Accreditation Cooperation (ILAC) published a document setting forth ILAC's policy regarding the requirements for the evaluation of the uncertainty of measurement in calibration and measurement, evaluation of the calibration and measurement capability (CMC), and the reporting of uncertainty on the certificates of calibration and measurement. It is applicable to calibration laboratories, reference measurement laboratories for laboratory medicine, and producers of certified reference materials that provide calibration and measurement services that refer to their accredited status under the ILAC Mutual Recognition Arrangement (MRA). Relevant sections of this policy may also be applicable to testing laboratories that perform their own calibrations. The policy becomes effective November 2011, that is 12 months from the release of the document in November 2010, and is available at http://www.ilac.org/documents/ILAC_P14_12_2010.pdf.

The policy is based on the "Guide to the Expression of Uncertainty in Measurement" (GUM, http://www.bipm. org/en/publications/guides/gum) and the ISO Guide 35 . The GUM describes an unambiguous and harmonised way of evaluating and stating the uncertainty of measurement and provides several options to estimate and state uncertainty of measurement. Similarly, ISO Guide 35 provides specific advice on determining the contributions to uncertainty from reference materials, including instability, inhomogeneity, and sample size, but several options are allowed. This may result in various interpretations of the GUM and ISO Guide 35, and hence calibration/reference measurement laboratories and reference material producers accredited by ILAC member bodies may report uncertainty of measurement in an inconsistent way. For this reason, many accreditation bodies, as well as regional co-operations, have published mandatory criteria documents and guidance on uncertainty of measurement, in line with the GUM and ISO Guide 35 to help laboratories implement the criteria and guidance.

Following a resolution approved at its 1999 General Assembly, ILAC developed requirements and guidelines for the estimation and statement of uncertainty in calibration and measurement, which apply to accreditation bodies and their accredited laboratories and reference material producers that perform calibration and measurement, in order to ensure a harmonised interpretation of the GUM and the consistent use of Calibration and Measurement Capability (CMC) by ILAC member bodies. This resulted in the recently released policy document that is in line also with the principles agreed upon between ILAC and the International Bureau of Weights and Measures (BIPM).

(source: http://www.ilac.org) 